\title{
Metodologías Activas para Alcanzar el Comprender
}

\author{
Arturo B. Rodriguez ${ }^{(1)}$, Leonardo J. Ramirez ${ }^{(2)}$ y Washington Fernández ${ }^{(3)}$ \\ (1) Univ. de Santiago de Chile, Depto. de Tecnología Industrial, Grupo de Investigación en Nuevas \\ Tecnologías (GINT), Santiago de Chile. (e-mail: arturo.rodriguez@usach.cl) \\ (2) Universidad Militar de Nueva Granada, División de Desarrollo Tecnológico e Innovación de la \\ Vicerrectoría de Investigaciones, Grupo de Investigación en Telemedicina (TIGUM), Bogotá, Colombia. \\ (e-mail: leonardo.ramirez@unimilitar.edu.co) \\ (3) Univ. del Bío-Bío, Depto. de Ingeniería Eléctrica, Concepción, Chile. (e-mail: wfernand@ubiobio.cl)
}

Recibido May. 23, 2016; Aceptado Jul. 26, 2016; Versión final Sep. 2, 2016, Publicado Feb. 2017

\begin{abstract}
Resumen
El artículo muestra los resultados de una intervención metodológica del tipo activa y cooperativa a través del uso de la tecnología robótica. El objetivo del estudio fue mostrar que las intervenciones n-dimensionales desarrollan el comprender sobre el hacer alcanzando aprendizaje significativo. Esta tecnología no se utiliza solo como elemento motivador y de entretenimiento, su uso cumple un papel protagónico dentro de la clase y se convierte en un instrumento del proceso enseñanza-aprendizaje. Las muestras corresponden a dos cursos, en dos semestres consecutivos, de tamaños poblacionales similares, grupo etario similar y distribución de género similar. Las variables definidas, registradas y analizadas son la comprensión y la dispersión en la sala de clase. Los resultados fueron significativos estableciéndose la importancia de lograr el hacer a través del camino impostergable del comprender.
\end{abstract}

Palabras clave: robótica; comprensión; proceso enseñanza-aprendizaje;dispersión

\section{Active Methodologies to Achieve Comprehension}

\begin{abstract}
The article shows the results of methodological intervention of the type active and cooperative through the use of robotic technology. The objective of the study was to demonstrate that $n$-dimensional interventions develop understanding over doing, allowing meaningful learning. This technology is not used only as a motivating and entertaining element; its use plays a leading role within the class and becomes an instrument of the teaching-learning process. The samples correspond to two courses, in two consecutive semesters, of similar population size, similar age group, and similar gender distribution. The variables defined, recorded, and analyzed are understanding and dispersion in the classroom. The results were significant, thus establishing the importance of achieving the "doing" through the unavoidable path of comprehension.
\end{abstract}

Keywords: robotics; comprehension; teaching-learning process; dispersion 


\section{INTRODUCCIÓN}

La Educación en los últimos diez años ha estado en el foco de atención de la población y del mundo político en Chile. Sin embargo, esta preocupación en el mundo docente investigativo data de algunos años antes. Temas como la deserción universitaria, calidad en la educación, modelos educativos, proceso enseñanza aprendizaje, estilos de aprendizaje, estilos cognitivos, metodologías activas y pasivas han sido y están siendo tratados en la literatura de hoy (Micari, et al., 2014; Haddix, 2014). La corriente pedagógica constructivista tan popular hoy, está basada en la teoría del conocimiento constructivista, que aborda el aprendizaje en el desarrollo de la autonomía del estudiante dando al docente un papel algo observador y poco interventor, de esta forma las estrategias son propias del estudiante. Pero esta construcción está orientada básicamente al Hacer, donde aparecen slogans muy difundidos como el "Aprender Haciendo", "Que el Aprendizaje Ocurra" entre otros. El hacer desde la perspectiva del equipo ha sido sobre valorado, dejando de lado el Comprender, se entiende que los estudiantes no solo deben saber hacer sino además deben comprender el fenómeno que observan, es decir deben tener la capacidad de clasificar, reconocer, sintetizar, reconocer, etc. Estos objetivos pocas veces se logran con el hacer, la capacidad reflexiva y codificadora de la realidad solo aparece a través de la comprensión (Tsai, Yea-Ru, 2016). Desde esta perspectiva, el equipo desarrolla un proyecto que permita observar si una intervención activa (desde el hacer) logra cambios significativos en el comprender. Para lograr que la intervención aglutine el interés y generé motivación se eligió la robótica como elemento interventor y el tema del enrutamiento en redes IP, este en particular es de difícil asimilación por incorporar muchos elementos matemáticos y algorítmicos, que hoy no generan convocatoria entre los jóvenes.

En la actualidad se utiliza la robótica como elemento motivador y no se tiene antecedente de una utilización como elemento educativo transversalizando con otras asignaturas. Por lo general, su utilización está circunscrita a la tecnología en sí misma, tales como levantar, desplazar objetos, baile robótico, etc. Sin embargo, las nuevas generaciones de estudiantes mantienen una relación "simbiótica" con la tecnología y todo aquello que no la involucre sencillamente es considerado "aburrido" (Austin et al.; 2016; Austin et al.; 2015. Lazzari, 2104). La dispersión en las clases que no utilizan tecnología en su composición áulica es mayor en las que si lo hacen. La situación actual de las asignaturas que involucran la enseñanza de las redes de datos, es la utilización de metodologías muy semejantes a las prácticas de la psicología conductista, basada en el proceso repetitivo hasta lograr su anclaje mejorando el proceso en cada repetición, dejando de lado el importante "comprender" a cambio de la urgencia del "hacer". En este sentido, la robótica tendrá un doble efecto en el aula, disminuirá la dispersión en la sala y aumentará la comprensión de los algoritmos de elevada abstracción. Al hacer visible la ejecución de los algoritmos y no quedarse sólo en un proceso matemático abstracto, la metodología aportará a la comprensión de los procesos algorítmicos. Mientras mayor sea el aprendizaje significativo de los procesos y conocimientos, mayor serán sus habilidades para modificarlo y proyectarlo para la creación de nuevo conocimiento, esto permitirá al estudiante generar autonomía.

El objetivo principal de la presente investigación, es la demostración que metodologías asociadas a procesos y/o actividades $n$-dimensionales, mejoran la comprensión generándose aprendizaje significativo en la sala de clase. Se espera aportar a la recuperación del "Cómo" y el "Por qué" como medio para llegar al "Hacer"; pero utilizando metodologías que persuadan sobre las metodologías que conducen; es decir hacer una revalorización del comprender (Rodríguez et al., 2014). La robótica es un tema actual y que llama la atención de los estudiantes, esto determinará una baja dispersión; además ayudará al docente a mejorar los niveles de comunicación interna en la sala y mantener focalizados a los estudiantes. Por otro lado las técnicas fenomenográficas de investigación ayudarán a describir la muestra y mostrar los avances logrado con la intervención en el aula (Tight, Malcolm, 2016; González., 2005; Rodríguez et al., 2015).

\section{REVISIÓN LITERARIA}

La intervención tecnológica en la sala de clases es de importancia para la educación de hoy, casos como la intervención que fue realizada para estudiantes discapacitados con mucho éxito, uso de tablets, utilización de computadores personales, entre otros. En el Congreso TEEM 2013 Technologycal Ecosystems for enhancing Multiculturality; se dedica un área a la robótica con el título "A robot in the classroom" (Track 6) con 12 tópicos, entre la que está "Teaching Methodology", esto muestra el importante enfoque que actualmente se le da a la tecnología robótica en otras partes del mundo. Por nuestros lares, sólo se utiliza la robótica como entretenimiento y motivación; dejando de lado su potencial como elemento metodológico (Eslava-Cobos et al., 2016).

El aporte de la robótica hoy no sólo se circunscribe a la solución de problemas mecánico-electrónicos sino al diseño de metodologías innovadoras que tienen como medio tecnológico la robótica para la obtención de un 
fin que no es necesariamente aprender robótica. Desde el punto de vista del conocimiento, los algoritmos de enrutamiento como Dijkstra, Floyd Warshall, etc., son de alta complejidad y la metodología utilizada sólo es de carácter conductista vía repetición de ejemplos, por lo que se reduce el objetivo sólo al "hacer" y no al "comprender" (Del Valle, et al., 2008).

En un sentido conductista desde la perspectiva del proceso, las redes neuronales funcionan basadas en caminos o conexiones asociadas a un peso; donde la repetición del evento refuerza el peso y la falta de evento debilita el peso (García, 2008); sin embargo los nuevos estudiantes expuestos a un elevado espectro tecnológico; son modulares y asocian los resultados como entradas a otro módulo para a su vez espera una respuesta que ingresará a otro módulo y así sucesivamente; son embriones de una cultura donde no importa el "Cómo" para ellos es más importante lo que sale. Por eso el estudiante cuando da "click" espera un resultado de esa acción, cuando no lo recibe siguen dando "click", luego si no funciona sólo apaga y enciende el computador, la tecnología que invade los hogares determina tarde o temprano su estilo de aprendizaje independiente del género afecta a todos por igual (Acevedo, 2015; Barragán, 2005; Carrasco, 1997; Díaz, 1999). Esa es la razón de la elevada dispersión en las salas donde existe ausencia de tecnología; la tecnología hoy debe ser una de los protagonistas del guion de la clase y no limitarse sólo a las presentaciones; las información debe tener n-dimensionalidad y la bidimensionalidad de la presentación no ayuda a la comprensión y a disminuir la dispersión en la sala de clases. La robótica tiene los elementos modulares que armonizan con el formato cognitivo del nuevo estudiante, por eso le llama la atención.

En la comunidad educativa se han utilizado las metodologías activas con la finalidad de mantener atento y focalizado al estudiante, que desarrolle trabajo en equipo y habilidades colaborativas. (Lazzari. 2014). La literatura muestra muchas variantes de esta metodología tales como el ABP o el aprendizaje basado en problemas, MdC o método de casos, ABPy o aprendizaje basado en proyectos y el aprendizaje colaborativo. Para el caso ABP, se realiza una investigación-diagnóstica tratando de detectar concepciones de los docentes y de los alumnos respecto de la resolución de problemas, que entrega un interesante resultado respecto que la efectividad de los métodos están fuertemente ligados a procesos que incentivan mecanismos de repetición y a acciones que validan este proceso resolutivo, acercándose más a una metodología conductista que constructivista (Del Valle et al., 2008); el caso MdC, es un método de aprendizaje basado en la participación activa, cooperativa y en el diálogo democrático de los estudiantes sobre una situación real. Consta de tres dimensiones, Papel Activo de los estudiantes; Nivel de cooperación de los estudiantes y la capacidad de dialogar para llegar a consensos (Asopa et al., 2001); el caso ABPy, el alumno pasa a ser el protagonista del proceso de enseñanza y aprendizaje y el profesor un mediador o guía de dicho proceso, proporciona a los estudiantes contextos de aprendizaje reales y los compromete en el diseño, la resolución del problema y, sobre todo, en la toma de decisiones y la actividad investigadora que conlleva, la diferencia más marcada con ABP es que puede tomar todo el semestre de estudio y a veces podría sobrepasarlo como continuidad del próximo (Austin et al., 2016); por último el aprendizaje colaborativo, es un sistema de interacciones cuidadosamente diseñado que organiza e induce la influencia recíproca entre los integrantes de un equipo.

\section{METODOLOGÍA}

El incremento de la comprensión de los algoritmos de enrutamiento, se espera a través de clases que permitan el trabajo en equipo y utilizando metodologías que involucren tecnología robótica; tratando de mantener focalizados a los estudiantes. Los algoritmos de enrutamiento tienen como objetivo la búsqueda de una ruta pasando por diferentes estaciones (routers); es análogo al "problema del viajero".

Para resolver este problema se adoptan diferentes algoritmos que permiten la obtención de dicha ruta. Por lo general, dicha solución pasa por un complejo algoritmo de pasos y secuencias que llevan a la solución, pero en el presente muchos de los estudiantes no logran comprender como es que logra obtenerse la ruta y como diferenciarlo de otros algoritmos que buscan lo mismo; y como consecuencia cuando utilizar un método u otro y cuando no; o bajo que escenarios de tráfico utilizarlos. Dicho objetivo no se logra con una instrucción plana bidimensional basada en la repetición del algoritmo hasta que logre hacerlo mecánicamente. El brazo del robot moviéndose en busca de la ruta pretende hacerlos entender porque es más lento en un caso y cuando es más rápido y debido a que escenario de tráfico se ralentiza.

Para ello se configurarán los brazos robóticos (kit Robobuilder) con 3 servomecanismos que permitan movimiento en el plano con posibilidad de una sola rotación. En la figura 1, se muestra el esquema de un brazo robótico con sus grados de libertad. Luego estudiantes programarán los brazos, para que se desplacen marcando cada nodo de la red, siguiendo un algoritmo especifico. Los movimientos rotacionales del brazo serán distintos y de acuerdo a las condiciones de tráfico establecidas. 


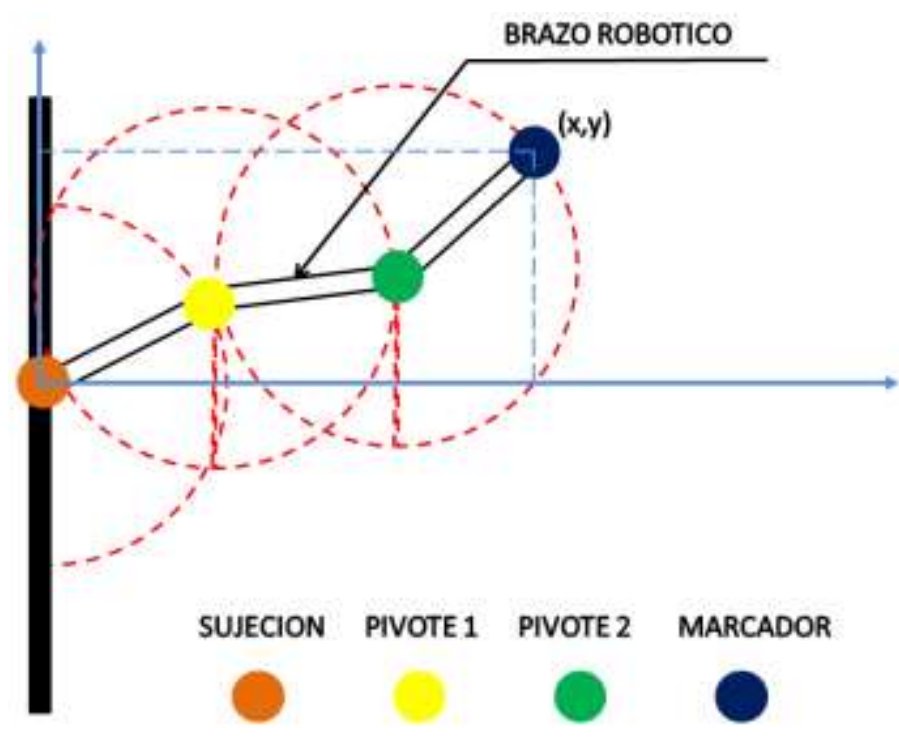

Fig. 1. Esquema del brazo robótico.

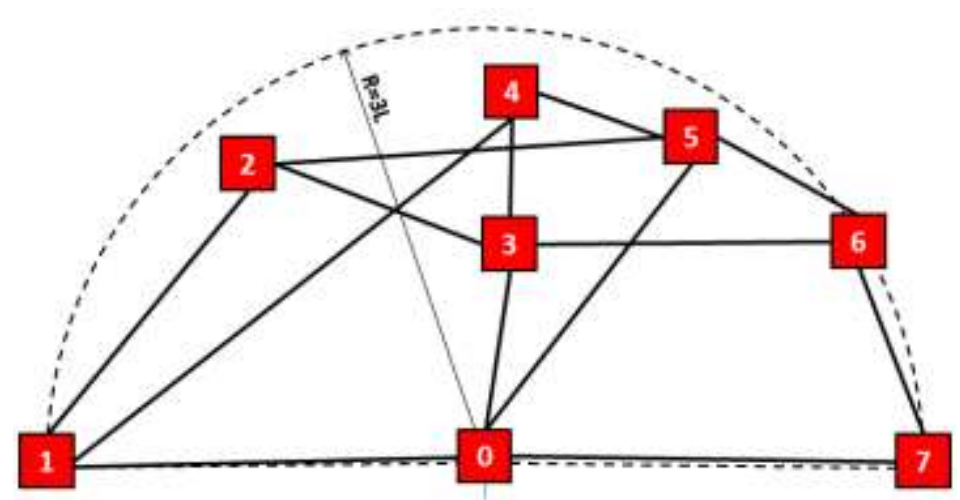

Fig. 2. Ejemplo de red dentro del alcance de los brazos $(R=3 L)$.

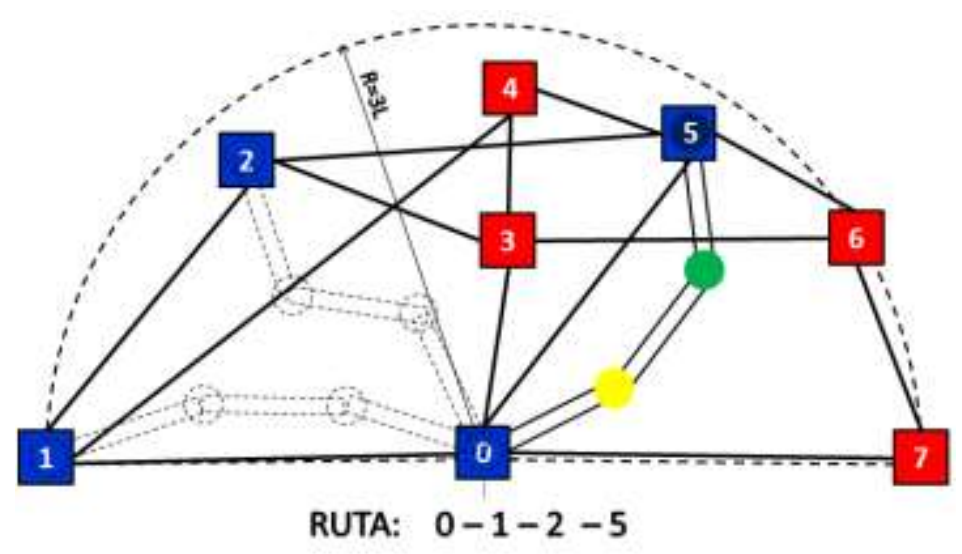

Fig. 3. Ejemplo de funcionamiento del brazo robótico recorriendo una ruta.

Los ensayos se realizarán en 4 grupos asociando diferentes algoritmos de enrutamiento cada vez, las evaluaciones antes y después del proceso serán realizadas por un evaluador externo al proyecto, mejorando la objetividad de los resultados. La metodología consta de 8 Etapas como se muestra en la figura 4. Se evaluarán los Indicadores asociados a la comprensión, Índice de Comprensión Antes (ICA) e Índice de Comprensión Después (ICD) de las actividades de Implementación y además el Índice de Dispersión en Sala antes y después de la clase (IDA e IDD). 


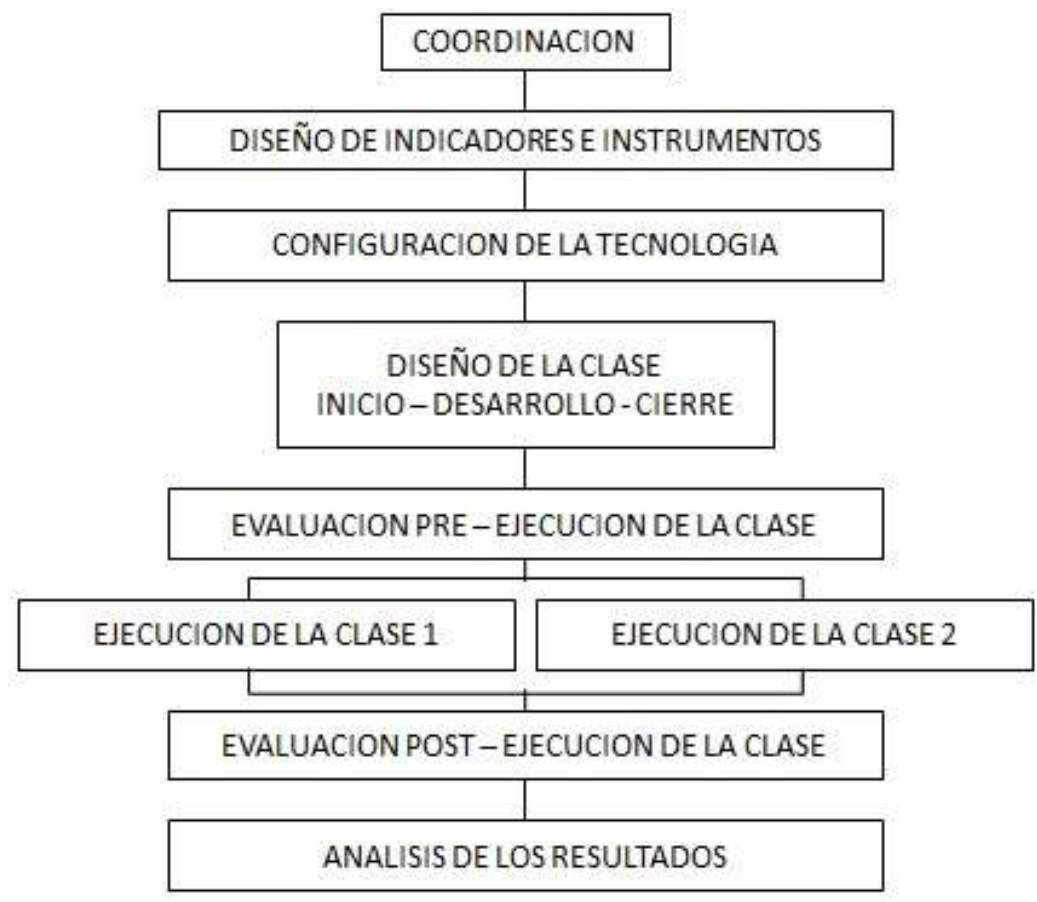

Fig. 4. Diagrama de flujo metodológico.

Índice de Comprensión (IC)

Para la medición del IC se elaboró un test de seis preguntas que indicarán el Concepto (CS), Selección (SA), Diferencia (DA), Tipos (TT), Clasificación (CA) y Reconocer (RT) el objeto de aprendizaje que en este caso fue el enrutamiento en las redes de datos. Las respuestas estaban en tarjetas y el estudiante debía seleccionar uno o varias de ellas, que se verificaban después con una rúbrica, no hubo interacción verbal entre evaluador y evaluado, las preguntas se realizaron individualmente (con cada estudiante) y mediante tarjetas, además se entrevistó antes y después de cada clase con evaluadores distintos en cada caso.

La clase 1 se llevó a cabo sin intervención tecnológica y con una clase intensamente instructivista y la clase 2 se llevó a cabo con una metodología activa y participativa con intervención de la tecnología robótica. Por ejemplo:

Pregunta uno: Seleccione los algoritmos convencionales del grupo de tarjetas (Ver Figura 5).

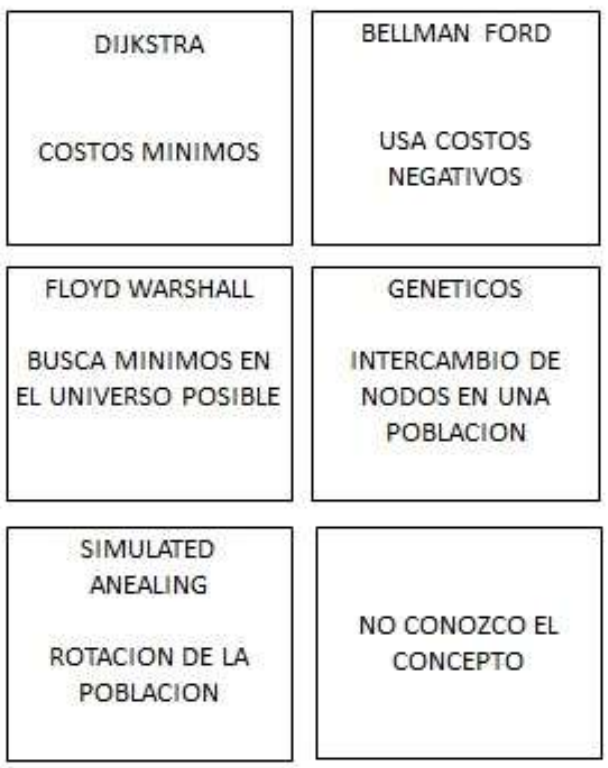

Fig. 5. Grupo de tarjetas para responder la pregunta de selección. 
Indicador de evaluación de la comprensión (IC), estableció según la rúbrica de cinco niveles donde 1 es la más baja y 5 es la más alta valoración, para cada pregunta. Se obtuvo el promedio del curso de esta valoración por cada pregunta y se obtuvo el ICD o ICA (2014-2 y 2015-1) según sea el caso, es decir antes y después de la intervención.

Se define:

$I C_{Y}^{Z}=\operatorname{Mod} a_{X=1}^{n} N C_{X-Y}^{Z}$

$I C_{Y}^{Z} \quad$ : Índice de Comprensión de la pregunta $Y$ del semestre $Z$.

$N C_{X-Y}^{Z}$ : Nivel de Comprensión del estudiante $\mathrm{X}$ de la pregunta $\mathrm{Y}$ del semestre $\mathrm{Z}$.

$n \quad:$ Número de estudiantes del curso, 21 y 19 para los semestres 2014-2 y 2015-1

$I C A_{Z}=\operatorname{Mod} a_{X=1}^{n} I C_{Y}^{Z}$

$I C D_{Z}=\operatorname{Mod} a_{X=1}^{n} I C_{Y}^{Z}$

$I C A_{Z}$ : Índice de Comprensión Antes de la intervención del semestre Z.

$I C D_{Z}$ : Índice de Comprensión Después de la intervención del semestre $Z$.

$N A P C_{Z}=I C D_{Z}-I C A_{Z}$

$N A P C_{Z}$ : Nivel de aumento de la Comprensión respecto de antes de la intervención del semestre Z.

Se evaluó, según la escala:

$N A P C=\{(0$, Mala $),(1$, Regular $),(2$, Aceptable $),(3$, Importante $),(4$, Destacable $),(5$, Excelente $)\}$

Índice de Dispersión (ID)

Para la medición del ID se necesitó la colaboración del docente y se dividió la sala en cuatro zonas (Ver Figura 6) dejando al docente la evaluación de la dispersión con una rúbrica de cinco niveles donde 1 es la más baja y 5 es la más alta valoración. El docente debió preocuparse que en cada zona hubiese la misma cantidad y los mismos estudiantes con la finalidad de no enrarecer la muestra en ambas clases (2014-2 y 2015-1). Indicador de dispersión (ID), el docente observo durante toda la clase y procedió a medir por zonas durante los primeros cinco minutos y durante los últimos cinco minutos de la clase.

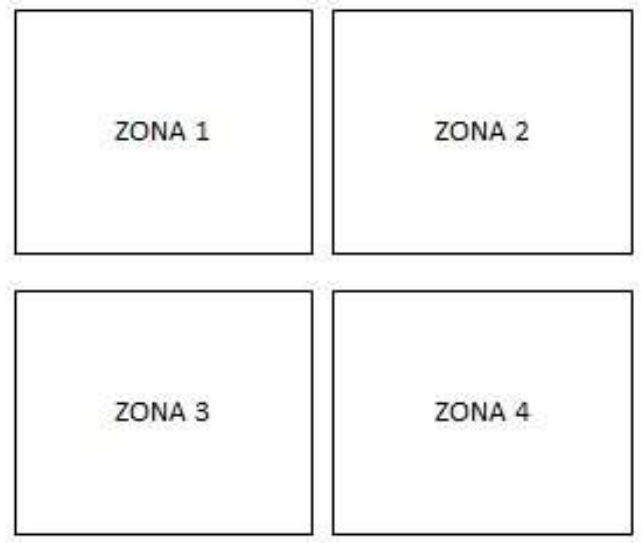

Fig. 6. Zonas de evaluación de la dispersión.

Se define:

$I D_{Y}^{Z} \quad$ : Índice de Dispersión de la Zona Y del semestre Z.

$I D_{Z} \quad$ : Índice de Dispersión del semestre $Z$.

$I D_{Z}=\frac{\sum_{Y=1}^{4} I D_{Y}^{Z}}{4}$ 
Además:

$N D D=\frac{I D_{2014-2}-I D_{2015-1}}{I D_{2014-2}} \times 100$

NDD : Nivel de aumento de la dispersión comparando ambos semestres.

Se evaluó, según la escala:
$N D D<10$
Mala
$10 \leq N D D<20$ Regular
$20 \leq N D D<30$ Aceptable
$30 \leq N D D<40 \quad$ Importante
$40 \leq N D D<50 \quad$ Destacable
$N D D \geq 50 \quad$ Excelente

\section{RESULTADOS Y DISCUSIÓN}

La asignatura en estudio se desarrolló en los semestres 2014-2 y 2015-1, en ambos semestres la edad promedio de los estudiantes es de 20 años. En la Tabla 1, se puede observar el tamaño de cada muestra y en la Tabla 2 se puede observar la distribución de género de las muestras. La distribución de género indica que tienen una distancia porcentual cercana y los estudiantes pertenecen al mismo grupo etario. La clase del semestre 2014-.2 utilizó una metodología instructivista totalmente clásica, el docente explicando y el estudiante escuchando. Mientras que la clase del 2015-1 utilizó una metodología activa con la intervención de la robótica como instrumento de aprendizaje.

Tabla 1. Muestra poblacional de cada clase.

\begin{tabular}{|c|c|c|}
\hline & $2014-2$ & $2015-1$ \\
\hline $\mathrm{N}$ & 21 & 19 \\
\hline
\end{tabular}

Tabla 2. Distribución de género de cada clase.

\begin{tabular}{|c|c|c|}
\hline & $2014-2$ & $2015-1$ \\
\hline$F$ & $4(19 \%)$ & $2(10,5 \%)$ \\
\hline$M$ & $17(81 \%)$ & $17(89,5 \%)$ \\
\hline
\end{tabular}

La Figura 7, muestra la comparación de los indicadores para cada pregunta, es evidente que la intervención metodológica determinó un cambio significativo respecto de las condiciones iniciales de los estudiantes respecto del tema a aprender (Enrutamiento). Por ejemplo para el caso de Selección algorítmica (SA) los estudiantes de la primera población lograron un incremento de un nivel mientras que para la segunda población el incremento fue de 3 niveles. Por lo demás, se logran incrementos significativos en el $85 \%$ de las acciones de comprensión, a excepción de la Diferencia de Algoritmos (DA) que no hubo diferencia.

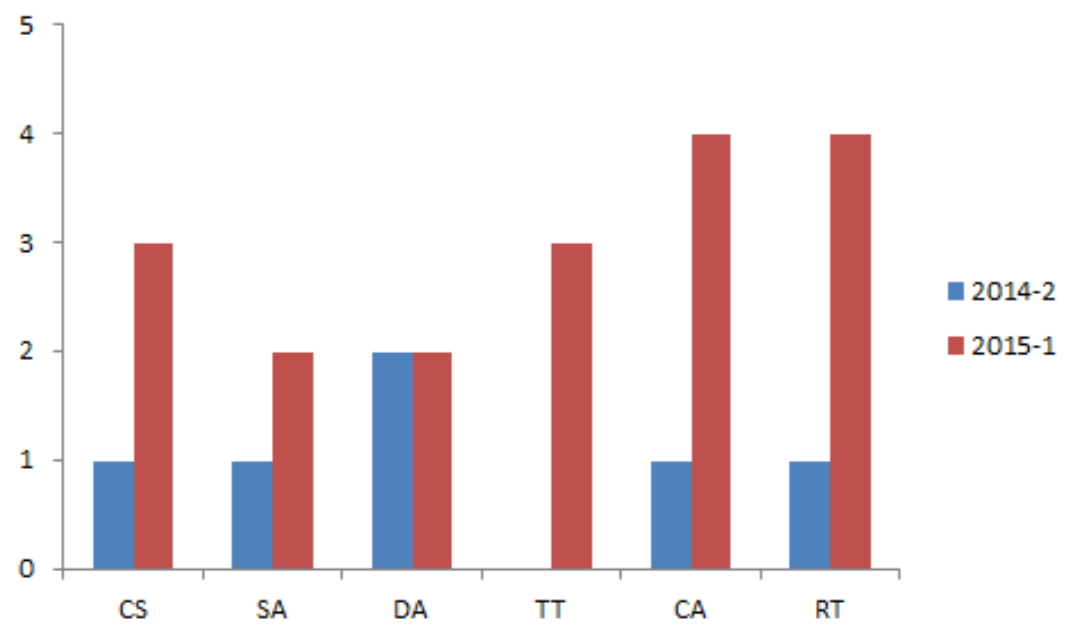

Fig. 7. Índices de comprensión antes y después de la intervención metodológica. Fuente: Propia 
El indicador de comprensión de la primera población (1 nivel) es inferior al indicador de comprensión de la segunda población (3 niveles) en dos niveles. La Tabla 3, muestra los indicadores de dispersión de las poblaciones en estudio, lográndose una disminución de la dispersión en un 54,4 \%.

Tabla 3. Zonas de evaluación de la dispersión. NDD=54.4\%

\begin{tabular}{|c|c|}
\hline $2014-2$ & 3,3 \\
\hline $2015-1$ & 1,5 \\
\hline
\end{tabular}

\section{CONCLUSIONES}

El uso de metodologías activas a través de una intervención metodológica con tecnología robótica muestra con claridad y cuantitativamente la mejora en los niveles de comprensión de las temáticas tratadas y la disminución significativa de los niveles de dispersión en la sala de clase. Se establece que en la presente intervención metodológica, mientras más tecnología se utilizó en la sala de clases mayor fue el interés que los estudiantes presentaron, esto ayudó significativamente al proceso de comprensión. Para lograr mayores precisiones se deberá proceder a realizar la intervención en diferentes semestres durante un periodo de tiempo mayor. Los alcances logrados con la aplicación metodológica están acordes con las componentes de las metodologías activas mostradas en la literatura como mejora del trabajo en equipo, mejoran las habilidades colaborativas y sobre todo los estudiantes son capaces de transmitir lo aprendido, esto es una consecuencia de la comprensión del fenómeno. Es de interés del equipo de investigación la de revalorizar la comprensión y utilizarlo como paso previo hacia el hacer, las metodologías constructivistas y conductistas muchas veces no logran controlar que esto suceda. El aprendizaje significativo mejora en la medida que somos capaces fomentar la comprensión en los estudiantes.

\section{AGRADECIMIENTOS}

Para la Vicerrectoría Académica de la Universidad de Santiago de Chile - USACH (Proyecto PID № 0182014), por su importante apoyo al desarrollo de la investigación y al Grupo de Investigación en Nuevas Tecnologías (GINT-DTI-USACH).

\section{REFERENCIAS}

Acevedo D., Cavadia S., Alvis A.,Learning Styles of Students of the Faculty of Engineering of the University of Cartagena (Colombia), Form. Univ. (en línea), 8(4), 15-22 (2015)

Anijovich, R. y Mora, S., Estrategias de enseñanza. Otra mirada al quehacer en el aula. Buenos Aires: Aique. (2009)

ASOPA, B. Y BEYE, G. Appendix 2: The case method. [Disponible en http://www.fao.org/docrep/W7500E/w7500e0b.htm] (2001)

Ausín V., Abella V., Delgado V. y Hortigüela D., Aprendizaje Basado en Proyectos a través de las TIC. Una Experiencia de Innovación Docente Ausín, Form. Univ. (en línea), 9(3), 31-38 (2016)

Ausín V., Abella V., Delgado V. y Hortigüela D., La Formación Docente y la Educación de Jóvenes y Adultos: Análisis de la Práctica Pedagógica para la Enseñanza de Ciencias, Form. Univ. (en línea), 8(1), 3$12(2015)$

García E., Neuropsicologia y Educacion. De las neuronas espejo a la teoría de la mente, Revista de Psicologia y Educacion. 1(3), 69-90 (2008)

Barragan, S., El Portafolio, metodología de evaluación y aprendizaje de cara al nuevo Espacio Europeo de Educación Superior. Una experiencia práctica en la Universidad de Sevilla. Revista Latinoamericana de Tecnología Educativa., 4.(1), 121-139 (2005)

Carrasco, J., Técnicas y recursos para el desarrollo de las clases. $3^{a}$ Ed. Ediciones Rialp. S.A. Madrid. España. (1997)

Del Valle, M., Curotto, M., La resolución de problemas como estrategia de enseñanza y aprendizaje. Argentina. Revista Electrónica de Enseñanza de las Ciencias, 7(2), 463-479 (2008) 
Díaz F., Hernández G., Estrategias docentes para un aprendizaje significativo. Una interpretación constructivista. México. McGraw-Hill (1999)

Eslava-Cobos, Jorge, y Ricaurte-Perdomo, Jennifer. Integración al Aula: Una Estrategia Para la Intervención y Evaluación, Guiada a Través del Uso de las Nuevas Tecnologías de Intervención (TICs). Psykhe, 16(2), 69-83 (2017)

González C., Díaz M. Aprendizaje colaborativo: una experiencia desde las aulas universitarias. Educación y educadores Universidad de la Sabana. Cundinamarca. Colombia, 8(1), 21-44 (2005)

Haddix, M-M., Generation Bullied 2.0: Prevention and Intervention Strategies for Our Most Vulnerable Students, Journal Of Adolescent \& Adult Literacy, 57(3), 250-252 (2013)

Lazzari M., Combinación de Aprendizaje Cooperativo e Individual en una Asignatura de Química de Materiales, Form. Univ., 7(4), 39-46(2014)

Micari M., Pazos P., Worrying about what others think: A social-comparison concern intervention in small learning groups, Active Learning In Higher Education, 15(3), 249-262(2014)

Rodriguez A., Cavieres E., Negrete C., DYNAMICS IN CLAss: An Intervention With Participatory Methodologies, EDULEARN14 Proceedings, 4401-4406 (2014)

Rodríguez A., Cavieres E., Negrete C. Participative Methodologies And Their Impact On Academic Performance, ICERI2014 Proceedings, 4232-4238 (2014)

Rodríguez A., Cavieres E., Rivera L., Ramirez L., Chahuan J. Methodological Intervention In Class: Learning From Comprehension, EDULEARN15 Proceedings, 1008-1012 (2015)

Tight, M., Phenomenography: the development and application of an innovative research design in higher education research, International Journal Of Social Research Methodology, 19(3), 319-338 (2016)

Tsai Y-R. ,Ouyang C-S., Chang Y-K., Identifying Engineering Students' English Sentence Reading Comprehension Errors: Applying a Data Mining Technique, Journal Of Educational Computing Research, 54(1), 62-84 (2016) 
